\title{
Dynamic characterization of meandering channels planform
}

\author{
Lucas Dominguez ${ }^{1}$, Ronald Gutierrez ${ }^{2}$, Yoch Ponte ${ }^{3}$, Jorge D. Abad $^{3, *}$, and Oscar Carrera ${ }^{3}$ \\ ${ }^{1}$ National University of the Littoral, Santa Fe, Argentina \\ ${ }^{2}$ Pontifical Catholic University of Peru, Lima, Peru \\ ${ }^{3}$ Water Research Center at University of Engineering and Technology, Lima, Peru
}

\begin{abstract}
Meandering channels develop different geometry patterns along the floodplain during its evolution. These natural processes lead to developing lateral and longitudinal migration, describing a sort of swept that affect the human activities. As the equilibrium geometry of natural channels depends on: the flow and sediment discharge, geological conditions of valley, soil properties, and vegetation among others, the preliminary characterization is not easy. Due to this, several engineering and scientific problems require an appropriate description of morphological processes. The dynamic characterization like the quantification of wavelength, amplitude, sinuosity, and curvature using Medium Temporal Scheme (MTS) is using widely. Unlike the traditional scheme that only uses short or long-term schemes, MTS includes relevant morphological events (e.g., cutoff). In addition this, the satellite images has increased and achieves a high spatial resolution, even more when studying large basins. This paper presents and validates a MATLAB ${ }^{\circ}$-based toolbox called Meander Statistics Toolbox (MStaT) to perform the dynamic characterization of several meandering channels using the MTS. To run MStaT only need the centerline (CL), and the average width of channel as input parameters. To maximize the toolbox, MStaT incorporates the Wavelet Analysis (WA) to decompose the signal (CL) and obtains the power spectrum. Finally, two studies cases (synthetic and natural channels) will be presented to validate MStaT.
\end{abstract}

\section{Introduction}

One of the structures commonly found in large alluvial basins are the meandering channels, characterized by presenting a high migration rate. This natural channel continuously modifies the fluvial environment, eroding the margin and affecting human activities [1]. Factors such as increases in sediment-laden flows, restoration of natural rivers, and construction or removal of water control structures, can affect the channels conditions. In the past, severe effects have been generated by the channelization, such as the increase on bed slope, change of flow velocity [2], or the sediment transport capacity, modifying the erosion or deposition process $[3,4]$. The principal ecology problems associate are the damage to habitat fish [5], and the reduction of the capacity of the stream on flood

\footnotetext{
* Corresponding author: jabadc@utec.edu.pe
} 
events [6]. All these effects must be understood and previously studied when designing an engineering project. It is ruled out that the resulting geometric condition for a given temporal state is the product of the hydrodynamic, and sediment transport. Due this, a preliminary description and quantification of the geometry parameters of a meandering channel are necessary to do.

The first criterion of meanders classification involving only the meander geometry is introduced by [7]. Then, [8] focus in a new description, which includes as the medium term coherent wave being present in the meander planform for a period that is strongly governed by the occurrence of cutoff events (medium-term frame). This new focus raised has a different position that the past research [7, 10], which used the centerline (short-term frame) to describe the morphodynamics.

On the other hand, the Wavelet Transform (WT) analysis has a high performance in different fields of application, since the bedforms description [9], and especially for the morphodynamics in meandering channels [8] with a quickly spectral analysis of signal. Doesn't exist yet a toolbox that uses a MTS [8] and generates a complete quantification of the geometry on meandering channels, even more, when talking about large channels.

This paper describes and validates the computation code called Meander Statistics Toolbox (MStaT) developed on MATLAB®. MStaT estimates geometry variables of the meanders like arc-wavelet, sinuosity, curvature, amplitude, inflection point, among others, and in turn perform a WT type analysis. This code represents a more effective performance of multi-disciplinary projects, such as restoring channelized streams to more natural, stable conditions or reducing the urbanization effect in waterways engineering. Different areas can use this code for each one. For example, the scientific utilities are: i) analyzing the interrelationship between meanders and planform, ii) the influence of sinuosity on the surface and groundwater interrelationship, or iii) impact of channelized in the meandering river. The engineering purposes are: i) the design of civil infrastructure protection, ii) river restoration, or iii) the design of waterways. Finally, a theoretical test and a natural channel to validate the code will be presented.

\section{Wavelet Analysis}

Previous research has represented the dynamics of the meandering rivers using different temporal schemes such as: 1) river centerline, short time scheme, or 2) the valley centerline, long time (geological scale). Both schemes offer different results depending on the dynamics of the channel $[10,11]$. [8] presented an intermediate temporal scheme, in which the centerline is defined between the loops of the meander, from here Mean Center (MC) line.

Wavelet Transforms (WT) play the main role for the MC determination, and have been successfully applied to other quasiperiodic geophysical signals such as bedform profiles [9]. The unidimensional WT of a signal (e.g., meander curvature, dependent on the longitudinal coordinate $s$ ) obtained by the convolution of the signal and the Wavelet Function (WF) or Mother Wavelet (MW) $\psi(s)$ as expressed by Eq. 1,

$$
T(a, b)=\int_{-\infty}^{\infty} x(s) \overline{\psi_{a, b}}(s) d s, \text { for } a>0
$$

where $a$ is the scale parameter, $b$ is the location parameter, and $\overline{\psi_{a, b}}$ is the complex conjugate of $\psi_{a, b}$ as mathematically represented by Eq. 2:

$$
\psi_{a, b}(s)=\frac{1}{\sqrt{a}} \psi\left(\frac{s-b}{a}\right)
$$


According to the Parseval's theorem [12] the same WT (Eq. 2) can be expressed by Eq. 3.

$$
T(a, b)=\frac{1}{2 \pi} \int_{-\infty}^{\infty} \hat{x}(f) \overline{\widehat{\psi_{a, b}}}(f) d f
$$

where $\hat{x}$ and $\widehat{\psi_{a, b}}(f)$ are the Fourier transforms of $x(f)$ and $\psi_{a, b}$, respectively. The wavelet power spectrum is defined as $|T(a, b)|^{2}$ and in terms of computational cost, it is less expensive to quantify it from Eq. 3 [13]. The continuous wavelet analysis is performed by Morlet wavelet function and modified version of the wavelet software provided by [13].

The Wavelet Principal Component Analysis (WPCA) plays a main role in the decomposition of the signal. WPCA uses two input parameters the discrete depth and the level $J$ at which the signal is filtered (with $J=1$ being the original signal). So, WPCA analyses of the signal at different frequencies with different resolutions to decompose the signal through a robust approximation and detailed coefficients. Daubechies algorithm is used to obtain the wavelet coefficient. Thus the composite of the larger scale is debunked through the filter [12]. For more details on the WPCA methodology, please refer to [14].

\section{MStaT Model Description}

MStaT was developed on MATLAB ${ }^{\circledR}$ to estimate the planar geometry, wavelet filter, and inflection point filter of meandering channels. MStaT was integrated into a Graphical User Interface (GUI) that allows seeing easily, interact with it, and interpret the data analyzed. The source codes are available for download at https://github.com/ldominguezruben/mstat. Also, an executable version is available at https://meanderstatistics.blogspot.com.ar/ with a quick start manual for its installation and execution with some study cases. Figure 1 shows a flowchart of MStaT model processes. Then will be detailed each step one.

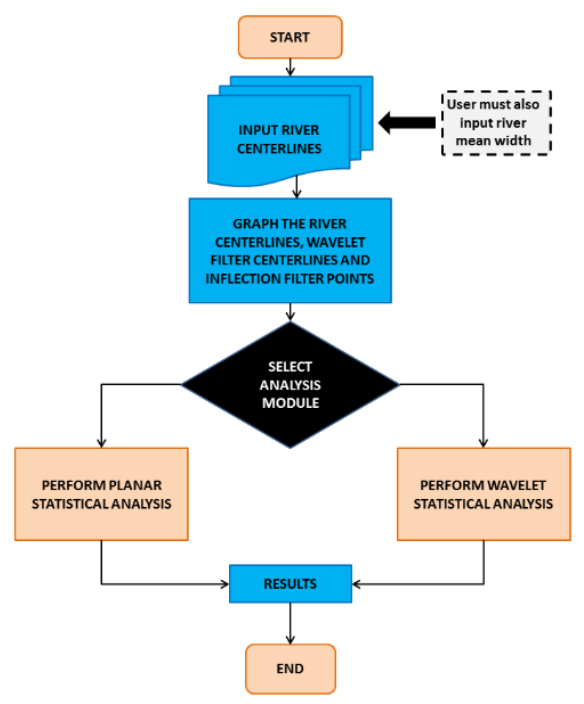

Figure 1. - Flowchart of MStaT computation code. 
Two input parameters are necessary to run MStaT. First the CL file, i.e., the $x$ and $y$ georeferencing points in a Coordinate System. Notice that $x$ and $y$ points are treated as imaginary numbers such as, the real part is the Easting, and the imaginary is the Northing. CL file must be prepared carefully in two-column $x$ and $y$ (without header). The formats accepted are .txt, .xlsx, .dat, or .kml (from Google Earth ${ }^{\circledR}$ ). To obtain these files it is possible to realize two digitalized methodologies details below.

The first method of digitization is through the use of ArcMap [15] (Figure 2). Herein will propose a methodology that uses the satellite images available from the United States Geological Survey (USGS) on its download website (https://earthexplorer.usgs.gov/). This website allows download of satellite images of different qualities and dates around the world. It is recommended to use the correct resolution depending on the scales of the channel. It is recommended to download satellite image with cloudiness of less than $20 \%$. So for this method will propose the next steps:

- Download the satellite image.

- Convert the Landsat image to water (black) and not water (white) using the Normalized Difference Vegetation Index (NDVI).

- Create a raster using ArcMap's Conversion Tools Toolbox, convert Raster to Polygon to create a shapefile of meandering channel.

- Using ArcMap's data Managment Tools Toolbox, convert Polygon to Line to create dual polylines of meandering channel. Use the Measure tool to calculate the average channel width (i.e. take minimum 30 samples).

- Using ArcMap's Cartography Tools Toolbox, Collapse Dual Lines to centerline to create meandering river centerline.

- Ensure the data points follow the downstream direction. Export the coordinates to a .txt file to be used in MStaT.

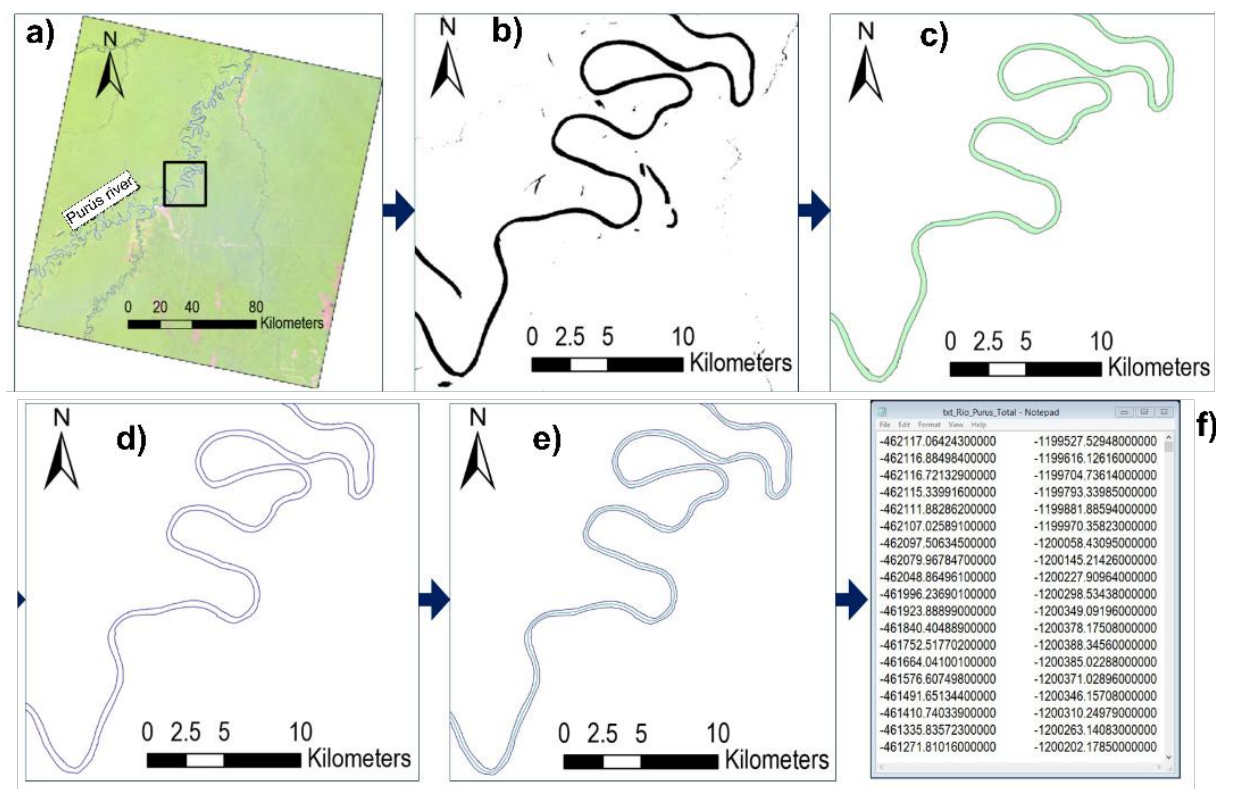

Figure 2.- Digitalized method of study reach. Method 1.

Second method possible is using Google Earth ${ }^{\circledR}$ application. MStaT users must generate a tools 'Add path' and perform the manual digitization of the channel. Once 
obtained it must save in. $\mathrm{kml}$ format. This method reduces the processing time with respect Method 1, due to the availability of high-resolution images offered by Google Earth ${ }^{\text {. }}$

Once digitalized the CL, the second input parameter is the average width of study reach. This parameter must be carefully chosen, as it will be of great use hereafter (the deviation can be less than 50\%). MStaT resampled the $x$ and $y$ data to get equally spaced points using as parameter the average width, and at the same time to convert the points of geographic data coordinates into local coordinates (e.g., $s$ the longitudinal coordinate and, $n$ the normal coordinate) using the [16] algorithm. Then, MStaT obtains the Mean Center (MC) line of the CL using the WPCA (see [14]). Herein the level used to decompose the CL is $J=6$ [8].

Subsequently, MStaT determinates the inflection points using the crossing between CL and MC (Mean Center Method, MCM), or the zero crossing points of CL (Inflection Point Method, IP). See Figure 2. Once identified bends by the method that the MStaT users chose (i.e., MCM or IP), calculates the planar geometry variables as maximum curvature, wavelength, sinuosity, amplitude and stream-wise orientation, among others (Figure 3).

Notice that MStaT determinates different classes of bends like upstream, downstream, symmetrical, and compound depending on the apex orientation. Another utility is the continuous Wavelet Analysis (WA) using the Morlet wavelet function. WA allows to obtain the wavelet spectrum of arc-wavelength $(\lambda)$ at $95 \%$ of confidence. This function is useful since it allows to identify the recurrence of $\lambda$ throughout the study reach. In the MStaT toolbar menu, the user can find the WA and obtains the wavelet spectrum and others parameters like curvature or normalized angle, and global wavelet spectrum for the normalized curvature of channel.

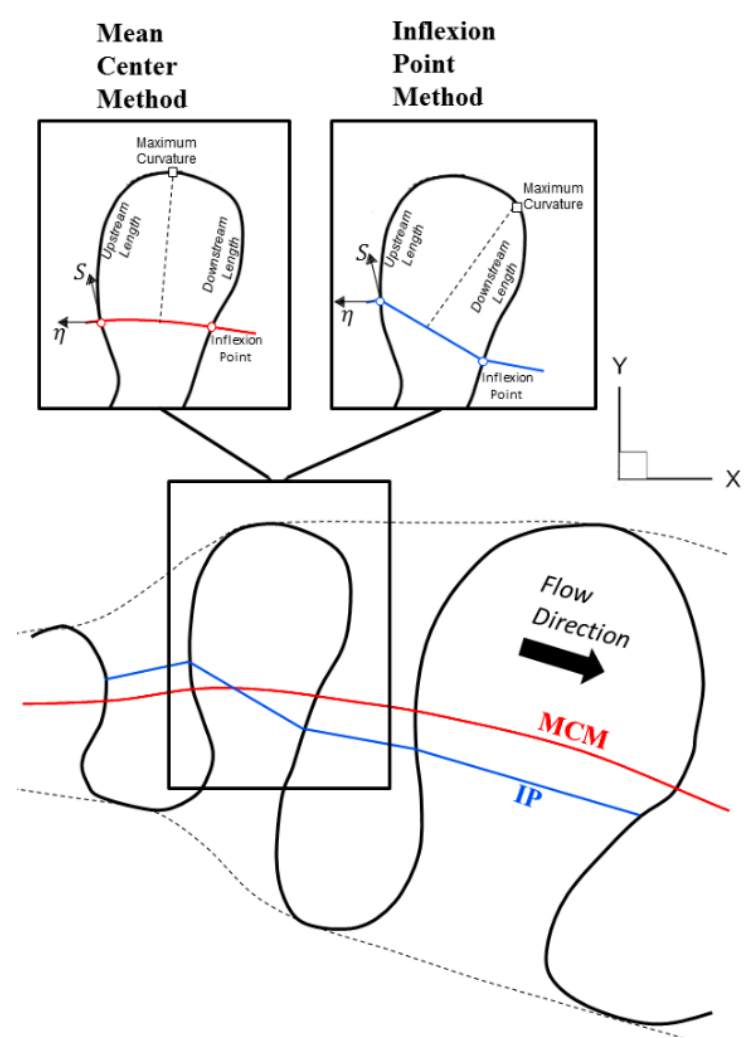

Figure 3. - Methods for the planar geometry analysis applied by MStaT. 


\section{Application and Validation}

By way of application and validation, 2 cases will be presented in this paper. The first is the Kinoshita equation [17] (synthetic meander), with the next parameters: $\lambda=25 \mathrm{~m}$ (arcwavelength), and $\theta=10$. See Figure 4: a,b,c,d,e.
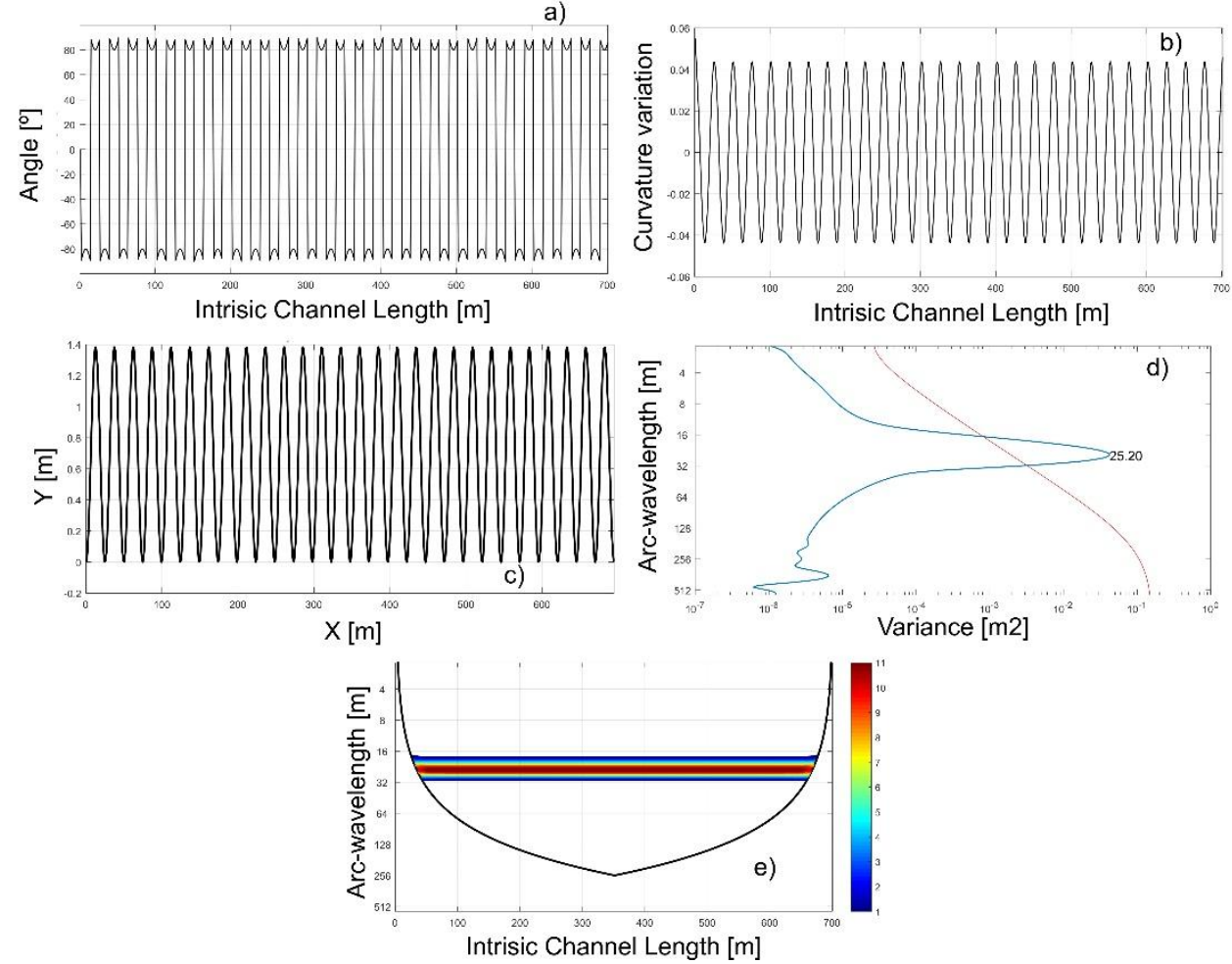

Figure 4.- Outputs graph from MStaT of synthetic meander channel (Kinoshita equation).

The second case corresponds to a natural channel, located in the Amazon basin of Peru (Corrientes River). For the latter, a total of $342 \mathrm{~km}$ was digitalized using the Google Earth ${ }^{\circledR}$ application. See Figure 5: a,b,c,d,e . In the case of the synthetic one, a great agreement between the $\lambda$ imposed (on the equation) and that obtained by the computational code is observed $(25.2 \mathrm{~m}$, differences $<0.8 \%)$. Besides, a clear monotonic arrangement of curvature and angle changes is observed for this case.

For the natural channel, its behavior is much less regular than the synthetic one, distinguishing greater changes in its curvature as $\lambda$ close to $2000 \mathrm{~m}$.

\section{Conclusion}

The knowledge of morphological variables in a meandering channel is critical when designing engineering projects. Understanding geometry variables of the channels allow the environment to be modified as little as possible. MStaT is an user-friendly toolbox developed on MATLAB $\AA$, which can aid designers and engineering with statistical analysis of meandering channels geometry. The easy-to-use GUI with graphical results represents a useful teaching tool for instructors of any skill level as well as an overview of 
key statistics for designers and consultants. This information is of great value when natural channels characteristics are compared to those in stream restoration or structural design. For example, river disturbances due to increased runoff from urbanization or the removal of aging dam structures could be quantified by the meander toolbox and mitigated properly due to an increased understanding of the migration process. Thus, MStaT represents a practical first step in description and characterization of meandering channels and understanding the environment impact.

a)
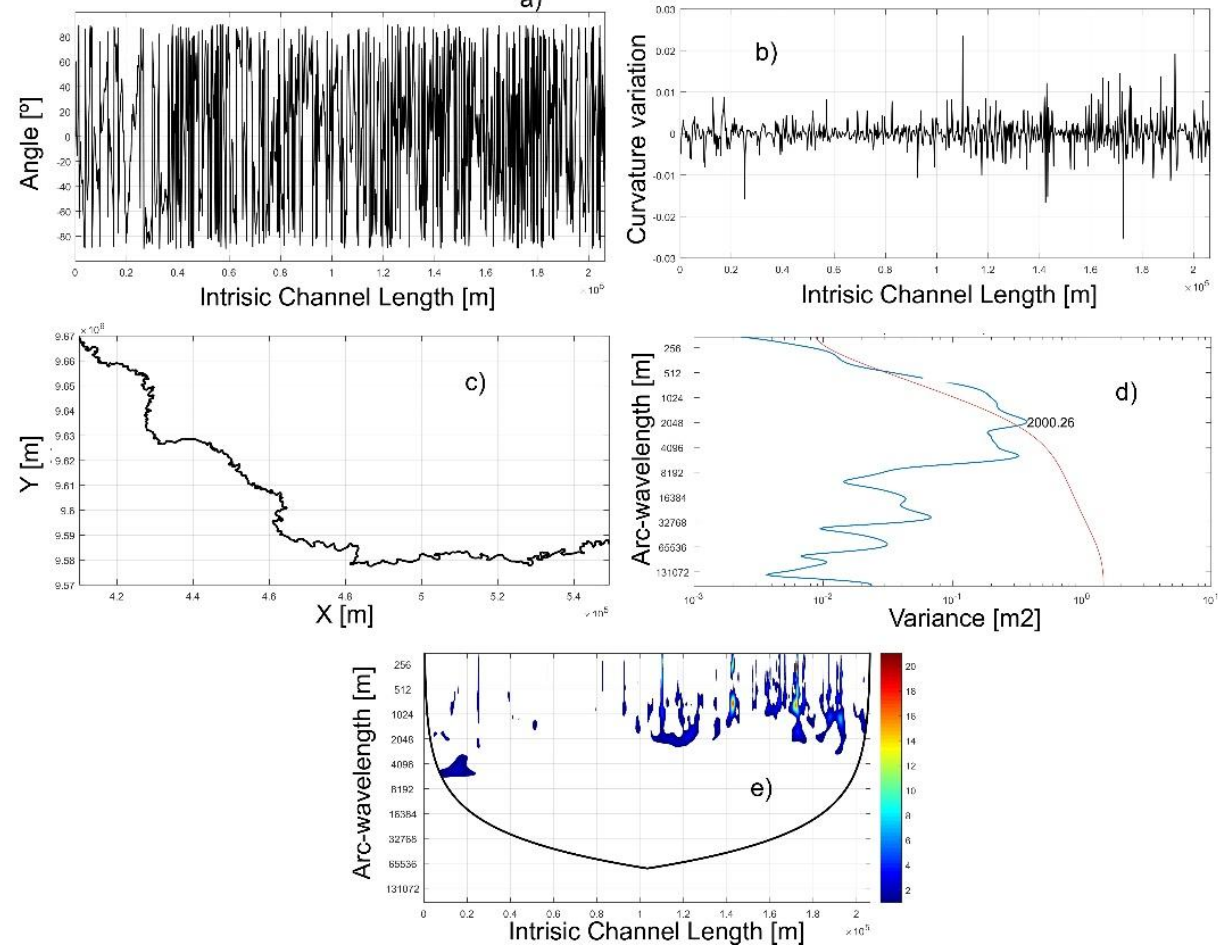

Figure 5.- Outputs graph from MStaT of natural meander channel (Corrientes River).

MStaT offers different capabilities such as:

- Visualize of river morphodynamics and planform migration.

- Calculate the planar statistics for individual river bends (number, sinuosity, orientation, wavelength, arc-wavelength, amplitude, downstream, and upstream length).

- Perform a wavelet filter analysis on the meander channels and outputs filtered results (curvature and angular amplitude).

- Classify meander channels for your shape (i.e., upstream, downstream, symmetric and compound).

Two studies cases corresponding to synthetic channels and natural channels were evaluated through this computation code, obtained a good performance $(<0.8 \%$, synthetic case). It also highlights the reduced time to evaluation of a large study reach like the Corrientes River.

Plans for MStaT includes an input of centerlines over time and calculation of river migration rates using curvilinear vectors. This would provide a more powerful estimate, which could be used in many applications in waterways engineering. In addition this, the existing code and data sets are in the process of rigorous testing and review as quality control. 


\section{References}

1. Güneralp, İ., and B. L. Rhoads (2011), Influence of floodplain erosional heterogeneity on planform complexity of meandering rivers, Geophys. Res. Lett., 38, L14401, doi: 10.1029/2011GL048134.

2. Hooke, J.M., (1984), Changes in river meanders, Progress in Physical Geography 8, 473-508.

3. Leopold, L. B., and M. G. Wolman, 1960. River Meanders. Bulletin of the. Geological Society of America. 71:769-794

4. Langendoen, and Simon, (2009), Modeling the evolution of incised streams. II: Streambank erosion, Journal of hydraulic engineering.

5. Kondolf, G. M., A. J. Boulton, S. O'Daniel, G. C. Poole, F. J. Rahel, E. H. Stanley, E. Wohl, A. Bång, J. Carlstrom, C. Cristoni, H. Huber, S. Koljonen, P. Louhi, and K. Nakamura (2006), Process-based ecological river restoration: visualizing threedimensional connectivity and dynamic vectors to recover lost linkages. Ecology and Society11(2): $5 . \quad$ [online] URL: https://www.ecologyandsociety.org/vol11/iss2/art5/

6. Hudson, P., and Kesel, R. (2000), Channel migration and meander-bend curvature in the lower Mississippi River prior to major human modification, Geology.

7. Brice, J. C. (1975), Air photo interpretation of the form and behavior of alluvial rivers, final report, U.S. Army Res. Off., Durham, N. C.

8. Gutierrez, R. R., Abad, J. D., 2014. On the analysis of the medium term planform dynamics of meandering rivers. Water Resources Research 50,3714-3733.

9. Gutierrez, R. R., J. D. Abad, D. Parsons, and J. Best (2013), Discrimination of bedform scales using robust spline filters and wavelet transforms: Methods and application to synthetic signals and the Rio Parana, Argentina, J. Geophys. Res. Earth Surf., 118, 1400-1418, doi:10.1002/jgrf.20102.

10. Abad, J. D., and M. H. Garcia (2006), RVR meander: A toolbox for re-meandering of channelized stream

11. Motta, D., J. D. Abad, E. J. Langendoen, and M. H. Garcia (2011), A simplified 2d model for long-term meander migration with physically based bank evolution, Geomorphology, 163-164, 10-25.

12. Addisson, P. (2004), The Illustrated Wavelet Transform Handbook, Inst. of Phys. Publ., Bristol, U. K.

13. Torrence, C., and G. P. Compo (1998), A practical guide to wavelet analysis, Bull. Am. Meteorol. Soc., 79, 61-78.

14. Aminghafari, M., N. Cheze, and J.-M. Poggi (2006), Least median of squares regression, Comput. Stat. Data Anal., 50, 2381-2398.

15. ESRI 2011. ArcGIS Desktop: Release 10. Redlands, CA: Environmental Systems Research Institute.

16. Leigleiter, C., and Kyriakidis, P.C. (2006), Forward and Inverse Transformations between Cartesian and Channel Fitted Coordinate Systems for Meandering Rivers, In Mathematical Geology Vol. 38, No. 8. doi: 10.1007/s11004-006-9056-6.

17. Abad, J. D., and M. H. Garcia (2009), Experiments in a high-amplitude Kinoshita meandering channel. 2: Implications of bend orientation on bed morphodynamics, Water Resour. Res., 45, W02402, doi: 10.1029/2008WR007017 